\title{
NF-kB expression in IgA nephropathy outcome
}

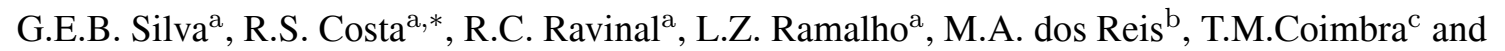 \\ M. Dantas ${ }^{\mathrm{d}}$ \\ ${ }^{\mathrm{a}}$ Department of Pathology, Faculty of Medicine of Ribeirão Preto, University of São Paulo, Ribeirão, Preto, Brazil \\ ${ }^{\mathrm{b}}$ Department of General Pathology, Faculty of Medicine of Triângulo Mineiro, Uberaba - MG. Brazil \\ ${ }^{\mathrm{c}}$ Department of Physiology, Faculty of Medicine of Ribeirão Preto, University of São Paulo, Ribeirão, Preto, Brazil \\ ${ }^{\mathrm{d}}$ Division of Nephrology, Faculty of Medicine of Ribeirão Preto, University of São Paulo, Ribeirão, Preto, Brazil
}

\begin{abstract}
Some studies have demonstrated the involvement of nuclear factor-kappa B (NF-kB) in the pathogenesis of glomerulonephritis. The aim of our study was twofold: (1) to analyze the prognostic value of NF-kB expression in primary IgA nephropathy (IgAN) and (2) to compare the results of NF-kB expression by immunohistochemistry (IHC) and southwestern histochemistry (SWH). We analyzed 62 patients diagnosed with IgAN from 1987 to 2003. We used monoclonal antibodies to CD68 and mast cell tryptase and polyclonal antibodies to TGF- $\beta 1, \alpha$-SMA and NF-kB p65. We used SWH for the in situ detection of activated NF-kB. The results showed that NF-kB expression (mainly by SWH) correlated with clinical and histological parameters. An unfavorable clinical course of IgAN was significantly related to tubular NF-kB expression by SWH, but not by IHC. The KaplanMeier curves demonstrated that increased NF-kB expression, which was measured by IHC and SWH, decreased renal survival. In conclusion, the increased expression of NF-kB in the tubular area may be a predictive factor for the poor prognosis of patients with IgAN. Compared with IHC, NF-kB expression determined by SWH was correlated with a larger number of parameters of poor disease outcome.
\end{abstract}

Keywords: IgA nephropathy, southwestern histochemistry, NF-kB, renal fibrosis

\section{Introduction}

IgA nephropathy (IgAN) remains the most common form of glomerulonephritis (GN) worldwide and may cause end-stage renal disease (ESRD) [2]. The progression of chronic renal failure in $\mathrm{GN}$ is characterized by the development of renal fibrosis. This process has been shown to be associated with phenotypic changes in glomerular and interstitial cells, and can be recognized by expression of smooth muscle actin ( $\alpha$-SMA) [1]. A large number of cytokines, inflammatory mediators and growth factors produced by macrophages and other cells, such as mast cells (MCs), are related to renal fibrosis $[4,6]$. Transcription factors, such as nucle-

* Address for Correspondence: R.S. Costa, Department of Pathology, Faculty of Medicine of Ribeirão Preto, Av. Bandeirantes 3900, CEP 14049-900, SP, Brazil. Tel./Fax: +55 (16) 3602 4551; E-mail: rscosta@fmrp.usp.br. ar factor-kappa B (NF-kB), have been shown to regulate many genes involved in the progression of renal disease [6].

Prior to activation, NF-kB remains in an inactive form in the cytoplasm, where it binds to inhibitory IkB proteins. The hallmark of NF-kB activation is the nuclear translocation of dimeric Rel protein transcription factors. Once activated, NF-kB, activates the transcription of target genes involved in inflammation, immunity, apoptosis, cell proliferation and differentiation [8, 16]. Using southwestern histochemistry (SWH), some previous studies demonstrated that NF-kB played a pivotal role in proteinuric nephropathy, such as minimal change disease, idiopathic membranous nephropathy, diabetic nephropathy and lupus nephritis [10,11,22]. Indeed, proteins have been shown to activate tubular expression of chemokines, mainly MCP-1, in proximal tubules through an NF-kB-dependent mechanism [6].

To understand the regulatory mechanism of gene expression, we used SWH to study in situ expression of 
activated NF-kB. In addition, we combined this method with standard immunohistochemistry (IHC). Our aim was to analyze whether the expression of NF-kB could be associated with inflammatory mechanisms and clinical parameters, which would predict an unfavorable renal clinical course in patients with IgAN. The results of the expression of NF-kB by SWH and IHC were also compared.

\section{Subjects and methods}

\subsection{Patients and clinical evaluation}

We studied renal biopsies from 62 patients with IgAN. Clinical and laboratory data were obtained from the medical records of the patients. The following classifications of IgAN outcome were considered during clinical follow-up [18]: (i) clinical remission (CR), which was proteinuria below $200 \mathrm{mg} /$ day with normal renal function; (ii) clinical improvement (CI), which was regression of nephrotic or nephritic syndrome or reduced proteinuria with stable renal function; (iii) unchanged (UC), which was unchanged clinical and laboratory data during the course of the disease, (iv) clinical worsening $(\mathrm{CW})$, which was aggravation of nephrotic or nephritic syndrome or increased proteinuria with normal renal function; and (v) chronic renal insufficiency (CRI), which was serum creatinine of $1.5 \mathrm{mg} / \mathrm{dL}$ or more and/or creatinine clearance of less than $90 \mathrm{~mL} / \mathrm{min}$ per $1.73 \mathrm{~m}^{2}$, or the need for dialysis or renal transplant (ESRD). We considered remission, clinical improvement and unchanged condition to indicate a favorable clinical outcome, whereas clinical worsening and chronic renal insufficiency indicated an unfavorable clinical outcome.

\subsection{Morphological evaluation}

The samples were analyzed using common light and immunofluorescence microscopy. The tissues obtained by renal biopsies from the 62 IgAN patients and control sections were stained with hematoxylin and eosin, Masson's trichrome and methenamine silver. Interstitial fibrosis was assessed in a semiquantitative manner: absent, $<10 \%$ of the total area; mild, 10 to $25 \%$ of the total area; moderate, 26 to $50 \%$ of the total area; and severe, $>50 \%$ of the total area. Frozen renal tissue specimens were prepared for immunofluorescence microscopy.

\subsection{Immunohistochemical studies}

This assay was performed according our previous description [18]. We used primary antibodies provided by Novocastra ${ }^{\circledR}$, Santa Cruz Biotechnology ${ }^{\circledR}$ and DAKO Corporation ${ }^{\circledR}$ : a polyclonal antibody for NF$\mathrm{kB}$ (1:200), a monoclonal mouse antibody for human macrophage CD68 (1:150), a monoclonal antibody for human MCs tryptase (1:30), a polyclonal antibody for human TGF- $\beta 1$ (1:250), and a monoclonal antibody for human $\alpha$-SMA (1:1000). Chromogen development was performed with 3,3' - diaminobenzidine (DAB) (Sigma), and the material was counterstained with methyl green prior to dehydration and mounting.

Quantification of MCs and macrophages was performed, and the number of cells $/ \mathrm{mm}^{2}$ was counted using a light microscope with a $10 \mathrm{X}$ magnification objective and an automatic image analyzer (KS 300 Kontron Imaging System, Eching bei München, Germany). Glomerular expression of $\alpha$-SMA and TGF- $\beta 1$ was graded semi-quantitatively [18]: 0 , no staining; 1 , trace mesangial staining; 2 , weak segmental mesangial staining; 3, strong segmental mesangial staining; and 4, strong diffuse mesangial staining. The $\alpha$-SMA and TGF- $\beta 1$ immunoreaction in the tubulointerstitium as well as the tubular NF-kB (SWH and IHC) of the renal cortex were scored as follows: 0 , absent staining; 1 , weak staining with focal distribution; 2 , moderate staining with focal distribution; 3 , strong staining with focal distribution or weak and diffuse; and 4, strong and diffuse.

\subsection{Southwestern histochemical analysis}

The non-radioactive in situ detection of NF-kB in paraffin-embedded kidney tissue preparations was performed by the SWH method, which has been previously described [8], using digoxigenin labeling and detection kits (Roche Applied Science, Indianapolis, USA). Synthetic sense DNAs (Imprit Genetics Corporation, Hialeah, USA), which contained the NF-kB sequence, were used as the probe. After annealing with the complementary sequence, the DNA probe was labeled with digoxigenin. The sections were then incubated with the labeled probes for $12 \mathrm{~h}$ at $37^{\circ} \mathrm{C}$. Slides were then incubated with an anti-digoxigenin antibody conjugated with alkaline phosphatase and detected using an NBT/BCIP solution. The slides were then mounted with glycerol. A mutant form of the probe labeled with digoxigenin was used as a negative control. 
Table 1

Clinical outcome of 62 patients with IgAN

\begin{tabular}{cc}
\hline Clinical outcome & $\mathbf{\%}$ \\
\hline Clinical remission(CR) & $7(11 \%)$ \\
Clinical improvement(CI) & $17(27 \%)$ \\
Unchanged(UC) & $4(7 \%)$ \\
Favorable course & $\mathbf{2 8}(\mathbf{4 5 \%})$ \\
Clinical worsening(CW) & $4(7 \%)$ \\
Chronic renal failure(CRF) & $12(19 \%)$ \\
End-stage renal disease(ESRD) & $18(29 \%)$ \\
Unfavorable course & $\mathbf{3 4}(\mathbf{5 5 \%})$ \\
\hline
\end{tabular}

\subsection{Statistical analysis}

When data distribution was normal, the values were expressed as mean \pm standard deviation. Proportions were determined using the standard chi-square test $\left(\chi^{2}\right)$ with Yates correction or Fisher's exact test. Correlations between non-continuous variables were calculated by Spearman's correlation coefficients. A significant difference was accepted if $p<0.05$.

Survival analysis was performed to test the association between renal failure and NF-kB (SWH and IHC). Survival times from the first clinical assessment to the last follow-up were obtained for each patient. Univariate comparisons of renal survival rates were made by Kaplan-Meier curves and the log-rank test.

\section{3. *Results}

Sixty-two patients were included in the present study: 32 men $(51.6 \%)$ and 30 women $(48.4 \%)$. The distribution of the 62 patients according to clinical outcome is shown in Table 1. For purposes of statistical analysis, we classified $28(45 \%)$ patients as having a favorable clinical course and $34(55 \%)$ patients as having an unfavorable clinical course. The average follow-up times were very similar between the two groups: 76.32 \pm 47.84 months for the patients with an unfavorable course and $73.29 \pm 50.85$ months for the patients with a favorable course.

\subsection{NF- $k B$ detected by $S W H$}

Using SWH, NF-kB was detected in cortical tubular epithelial cells and sparsely in some glomerular cells. This pattern of activated NF-kB was observed in the renal sections of most IgAN patients, but it was absent in the normal renal specimens of the controls (Fig. 1).

There were significant correlations between the histological scores for NF-kB expression measure by SWH in the tubular area and the magnitude of protein-
Table 2

Clinical outcome of 62 IgAN patients as a function of NF-kB by SWH score in tubular area

\begin{tabular}{cccccccc}
\hline NF-kB SW & \multicolumn{6}{c}{ Clinical outcome } & Total \\
\cline { 2 - 7 } Score & CR & CI & UC & CW & CRI & ESRD & \\
\hline $\mathbf{0}$ & 4 & 1 & 9 & 1 & 2 & 1 & 18 \\
$\mathbf{1}$ & 2 & 2 & 6 & 3 & 6 & 12 & 31 \\
$\mathbf{2}$ & 1 & 1 & 2 & 0 & 4 & 5 & 13 \\
Total & 7 & 4 & 17 & 4 & 12 & 18 & 62 \\
\hline
\end{tabular}

Legend: CR, Clinical remission; CI, Clinical improvement; UC, Unchanged; CW, clinical worsening; CRI, Chronic renal insufficiency; ESRD, end-stage renal disease.

Comparing $(\mathrm{CR}+\mathrm{CI}+\mathrm{UC}$ and $\mathrm{CW}+\mathrm{CRI}+\mathrm{ESRD})$ versus $(0$ and $1+2): \chi^{2}$ Test, $p=0,003$.

uria at the time of biopsy $(p=0.02)$ and at the end of the follow-up $(p<0.01)$. There were also correlations between NF-kB expression measured by SWH and serum creatinine at the time of biopsy and at the end of the follow-up $(p<0.01)$. Similarly, significant correlations were found between the same scores for tubular NF-kB expression measured by SWH and the presence of arterial hypertension at the time of biopsy and at end of the follow-up ( $p<0.01)$.

We observed a significant correlation between tubular NF-kB expression and the clinical course (Table 2). Indeed, the absence of NF-kB expression (SWH score $0)$ indicated a favorable clinical course $(\mathrm{CR}+\mathrm{CI}+\mathrm{UC})$ and the presence of NF-kB expression (SWH score 1 or 2) was correlated with an unfavorable clinical course $(\mathrm{CW}+\mathrm{CRI}+\mathrm{ESRD})(p=0.01)$.

Tubular activated NF-kB expression was correlated with the degree of tubulointerstitial lesion and the presence of vascular injury $(p<0.01)$. There were no significant correlations between tubular activated NF$\mathrm{kB}$ expression measured by $\mathrm{SWH}$ and interstitial and glomerular TGF- $\beta 1$ or glomerular and tubulointerstitial $\alpha$-SMA expression. The expression of NF-kB measured by SWH did not show a positive correlation with the infiltration of MCs $(p=0.11)$. It did tend to correlate with macrophages in the interstitial compartment $(p=0.07)$.

\subsection{NF- $k B$ detected by IHC}

Using IHC, normal epithelial tubular cells in control sections demonstrated weak cytoplasmic staining for NF-kB, which was regarded as the baseline staining. Most of our IgAN cases showed stronger cytoplasmic staining than baseline (sometimes focal or diffuse). No nuclear staining was observed in the present study, and some slides were also prepared without counterstain to prove that (Fig. 1). 

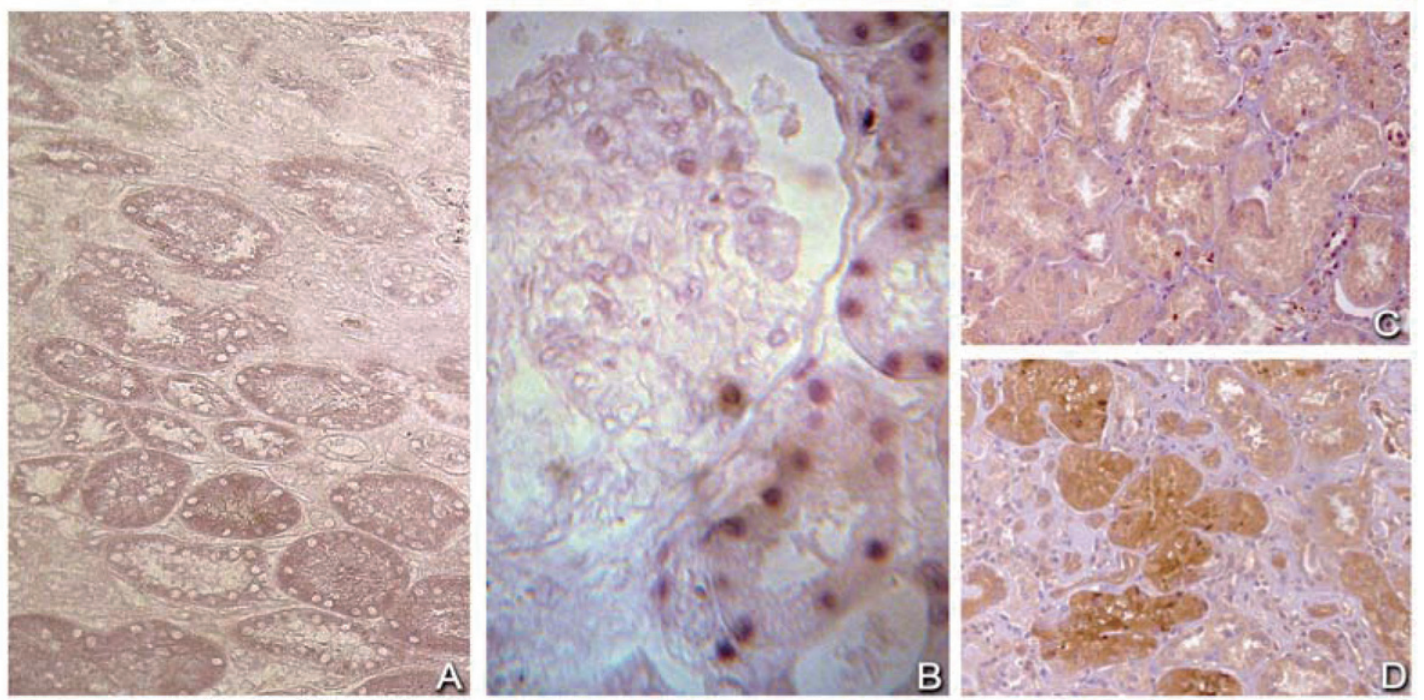

Fig. 1. Southwestern histochemistry (SWH) and Immunohistochemistry (IHC) for NF-kB. SWH: (A) the absence of staining in a preserved area of cortex, (B) moderate staining in tubules and weak staining in the glomeruli. IHC: (C) the baseline staining in preserved areas of the renal cortex, (D) foci of strong staining in preserved tubules in areas of interstitial fibrosis (A: objective 10x; B, C, D: objective 40x). )

Table 3

Clinical outcome of 62 IgAN patients as a function of NF-kB IHC score in tubular area

\begin{tabular}{|c|c|c|c|c|c|c|c|}
\hline \multirow{2}{*}{$\begin{array}{c}\text { NF-kB IHC } \\
\text { Score }\end{array}$} & \multicolumn{6}{|c|}{ Clinical outcome } & \multirow[t]{2}{*}{ Total } \\
\hline & $\mathrm{CR}$ & $\mathrm{CI}$ & $\mathrm{UC}$ & $\mathrm{CW}$ & CRI & ESRD & \\
\hline 0 & 2 & 1 & 2 & 1 & 2 & 0 & 8 \\
\hline 1 & 3 & 1 & 8 & 3 & 2 & 4 & 21 \\
\hline 2 & 2 & 2 & 6 & 0 & 7 & 12 & 29 \\
\hline 3 & 0 & 0 & 1 & 0 & 1 & 2 & 4 \\
\hline Total & 7 & 4 & 17 & 4 & 12 & 18 & 62 \\
\hline
\end{tabular}

Legend: CR, Clinical remission; CI, Clinical improvement; UC, Unchanged; CW, clinical worsening; CRI, Chronic renal insufficiency; ESRD, end-stage renal disease.

Comparing $(\mathrm{CR}+\mathrm{CI}+\mathrm{UC}$ and $\mathrm{CW}+\mathrm{CRI}+\mathrm{ESRD})$ versus $(0+1$ and $2+3$ ): Fisher exact test, $p=0,17$.

There were significant correlations between histological scores for NF-kB expression by IHC in the tubular area and both the magnitude of proteinuria at the end of the follow-up $(p=0.01)$ and serum creatinine at the time of biopsy $(p<0.01)$. There were not significant correlations, however, between NF-kB expression, determined by IHC, and urinary protein excretion at the time of biopsy ( $p=0.32$ ) or serum creatinine at the end of the follow-up $(p=0.28)$. Although there was not a significant correlation between NF-kB scores measured by IHC and the presence of arterial hypertension at the time of biopsy ( $p=0.45)$, there was a correlation between NF-kB and the presence of arterial hypertension at end of the follow-up $(p<0.01)$. No significant correlations were observed between clinical course and NF-kB expression measured by IHC (Table $3, p=0.28$ ).

Tubular NF-kB expression measured by IHC was correlated with the degree of tubulointerstitial fibrosis and the presence of vascular injury $(p<0.01)$. There were no significant correlations, however, between tubular NF-kB measured by IHC and interstitial and glomerular TGF- $\beta 1$ or glomerular and tubulointerstitial $\alpha$-SMA expression. The expression of NF-kB measured by IHC showed positive correlations with the infiltration of MCs and macrophages in the interstitial compartment ( $p=0.01$ and $p<0.01$, respectively). The Spearman correlation coefficients between tubular expression of NF-kB (SWH and IHC), and both interstitial MCs and macrophages are shown in Fig. 2.

Figure 3 shows that the expression of NF-kB, measured by both IHC and SWH was correlated with renal survival in subsequent months, which was demonstrated by the Kaplan-Meier curves. After analysis with the log-rank test, high scores for NF-kB measured by both IHC and SWH predicted poorer survival of renal function $(p<0.01)$.

\section{Discussion}

Mezzano et al. detected NF-kB expression, measured by $\mathrm{SWH}$, in cortical tubular epithelium and sparsely in podocytes of a few glomeruli. IHC was also able to detected NF-kB in tubular epithelial cells (cytoplasm 

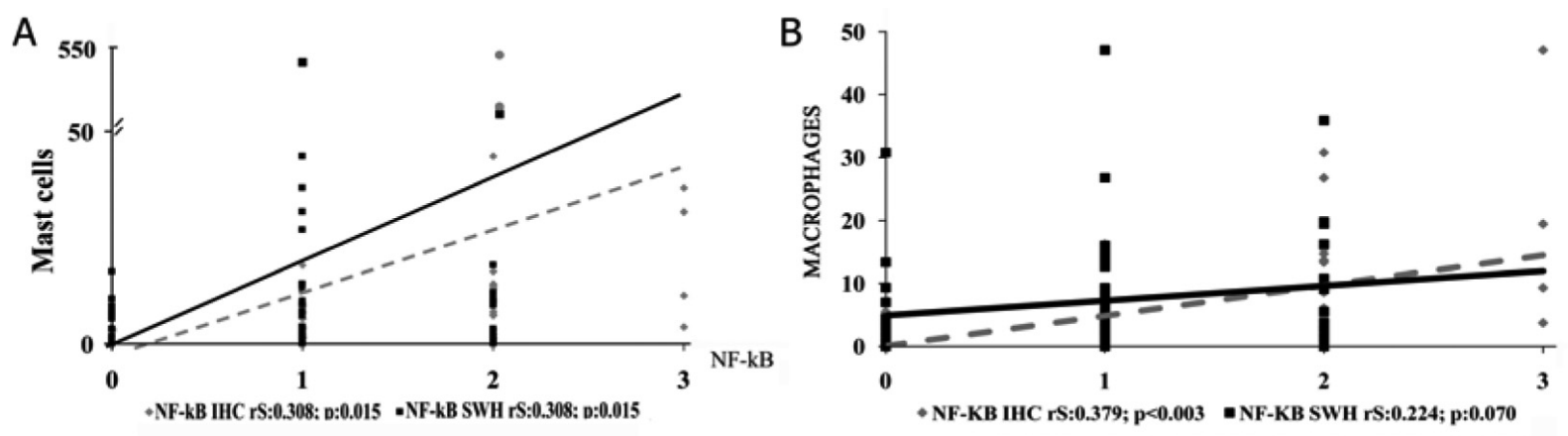

Fig. 2. (A) Spearman's correlation coefficients (r) between MCs and NF-kB measured by SWH and IHC; (B) Spearman's correlation coefficients (r) between macrophages and NF-kB measured by SWH and IHC.
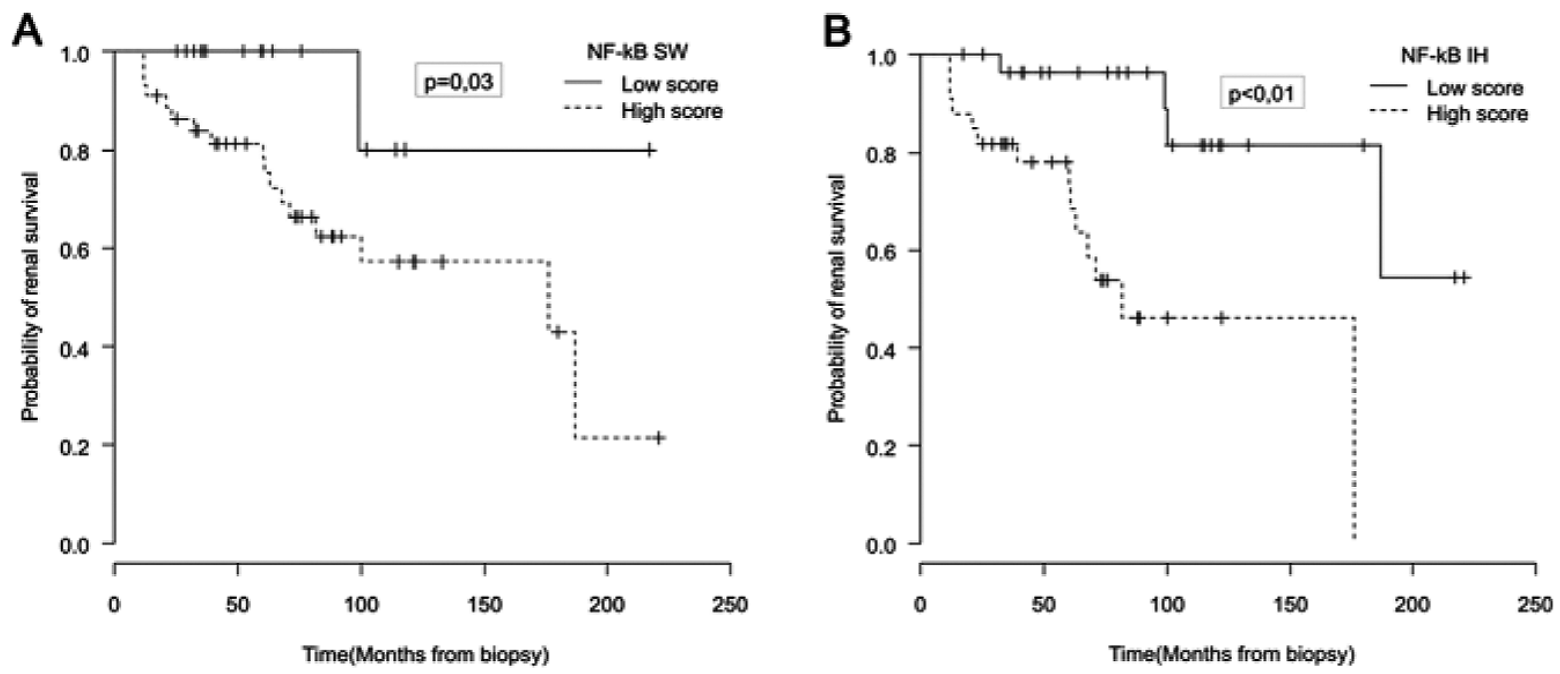

Fig. 3. This figure shows the renal survival of patients with $\operatorname{IgAN}(n=62)$ as a function of NF-kB measured by IHC and SWH. The score of 0 or 1 was considered to be low, and a score of 2 or 3 (not found in NF-kB measured by SWH) was considered to be high. The end point for renal survival was defined as ESRD requiring dialysis or transplant. Vertical hatched marks on the survival curves indicate the end of follow-up for patients who did not develop ESRD.

and nucleus) $[10,11]$. The use of SWH in the present study showed a distribution of NF-kB staining similar to that described above. For IHC, staining was only found in the cytoplasm, which means that NF-kB was in an inactive form. Activation of NF-kB in renal tubular cells has been implicated in tubulointerstitial injury in a proteinuria-induced model in rats [15]. In a study of various human glomerulopathies, including $\operatorname{IgAN}$, the expression of NF-kB, measured by $\mathrm{SWH}$, was mainly present in patients with proteinuria [10]. These findings were similar to those from our study, which found a positive correlation between initial proteinuria and NF$\mathrm{kB}(\mathrm{SWH})$ and final proteinuria and NF-kB (SWH and $\mathrm{IHC})$.

In models of kidney disease caused by tacrolimus, the activation of NF-kB was increased, and it was as- sociated with decreased renal function and increased tubulointerstitial injury [19]. In vivo and in vitro studies have suggested that the transcription factor NF-kB plays a role in tubulointerstitial injury. Indeed, both IHC and SWH demonstrated that the activation of NF$\mathrm{kB}$ was related to tubulointerstitial injury and deterioration of renal function $[3,22]$. These findings were similar to those from our study, which found positive correlations between NF-kB expression (SWH and IHC) and serum creatinine at the time of biopsy as well as NF-kB (SWH) expression and serum creatinine at the end of the follow-up. In experimental GN, the expression of NF-kB was higher in areas where there were increased numbers of macrophages and myofibroblasts [8]. These data suggested that activation of interstitial fibroblasts was attenuated by inhibition 
of NF-kB in this experimental model. In the present study, there was no correlation between the expression of glomerular or tubulointerstitial $\alpha$-SMA and tubular $\mathrm{NF}-\mathrm{kB}$ (SWH and IHC). It is important to remember that $\alpha$-SMA is only expressed in the early stage of fibrosis, and cases with more favorable courses of IgAN were not present in our series.

In rats, the suppression of endothelial NF-kB, measured by IHC, showed a decrease in renal injuryinduced arterial hypertension [7]. In human renal disease, there has not been any histologic evidence of NF$\mathrm{kB}$ activation in vascular cells $[10,11,17,22]$. Although we also failed to find vascular expression of NF-kB by SWH or IHC, our study showed that NF-kB, measured by both SWH and IHC, was correlated with the presence of vascular injury. In addition, we observed positive correlations between hypertension at the time of biopsy and NF-kB expression by SWH as well as hypertension at the end of follow-up and NF-kB expression by SWH and IHC. The correlation between vascular injury and tubular NF-kB expression can be explained because NF-kB activation has been demonstrated in all phases of renal disease, even in late stage of diseases, which there is hypertension and vascular injury [17].

Transcription factors have been shown to induce the activation of TGF- $\beta$ [24,33]. Indeed, the activation of NF-kB has been demonstrated to be concomitant with the expression of proinflammatory (MCP-1, RANTES, OPN) and pro-fibrogenic genes (TGF- $\beta$ ) [10]. These data supported the theory that NF-kB induces activation of proinflammatory and profibrogenic genes. In our patients, we did not found a correlation between glomerular or tubulointerstitial TGF- $\beta$ and NF-kB expression (SWH and IHC). These results may be due to the low number of cases expressing TGF- $\beta$, but it is also possible that IHC was not sensitive enough to evaluate renal TGF- $\beta[5,18]$.

Several studies have shown the involvement of inflammatory cells in renal fibrosis. One study showed a correlation between the activation of NF-kB and interstitial infiltration of macrophages in lupus nephritis [22]. In addition, recent studies have suggested that the signaling pathway of NF-kB was involved in the activation of macrophages [21]. In our study, we only found a correlation between macrophage infiltration and NF-kB measured by IHC. Although the role of $\mathrm{NF}-\mathrm{kB}$ in the activation of mast cells has been demonstrated, none of these studies examined this relationship in renal disease [12]. We found a correlation between NF-kB, measured by IHC, and the number of MCs infiltrating the interstitium of patients with IgAN.
NF-kB participates in the regulation of gene transcription by binding to a specific sequence of nuclear DNA known as a response element. To better understand the operation of this transcriptional factor, an analysis of the expression at the individual cell level is required. In IHC, NF-kB was detected whether or not it was bound to the response element. In SWH, however, NF-kB was only detected when it was unbound or weakly bound to the response element. Because these methods provide complementary results to each other, they can be used to characterize the physiological state of NF-kB [9]. In our study, there was no correlation between SWH and IHC, which can be explained by the fact that IHC and SWH detect different forms of NF-kB. Moreover, NF-kB is a family that integrates an intricate network of signaling pathways, and the functional consequences of this variability are not fully understood. None of the techniques used in the literature offer a fully comprehensive description of NF-kB activation and function [17].

In conclusion, renal biopsies of patients with IgAN showed that NF-kB expression evaluated by SWH correlated with a higher number of parameters of poor prognosis than NF-kB expression measured by IHC. However, NF-kB detected by IHC showed more significant correlations with inflammation (macrophages and mast cells), which is a key factor in the progression of glomerulopathies. The differences between the two techniques reflected the variability and complexity of NF-kB action in human renal injury [13]. Therefore, they are complementary techniques and both serve as markers of IgAN disease progression.

\section{Acknowledgements}

R.S. Costa, M.A. dos Reis, M. Dantas and T.M. Coimbra were recipients of CNPq fellowships. This research was partially supported by FAPESP.

\section{References}

[1] C.E. Alpers, K.L. Hudkins, A.M. Gown and R.J. Johnson, Enhanced expression of "muscle-specific" actin in glomerulonephritis, Kidney Int 41 (1992), 1134-1142.

[2] F.C. Berthoux, H. Mohey and A. Afiani A, Natural history of primary IgA nephropathy, Semin Nephrol 28 (2008), 4-9.

[3] J.C. Costa, R.S. Costa, C.G. Silva and T.M. Coimbra, Enalapril reduces the expression of nuclear factor-kappaB and cJun N-terminal kinase in the renal cortices of five-sixthsnephrectomized rats, Am J Nephrol 26 (2006), 281-286.

[4] T. Ehara and H. Shigematsu, Mast cells in the kidney, Nephrology (Carlton) 8 (2003), 130-138. 
[5] D.S. Goumenos, A.C. Tsamandas, S. Oldroyds, F. Sotsiou, S. Tsakas, C. Petropoulou, D. Bonikos, A.M. El Nahas and J.G. Vlachojannis, Transforming growth factor-beta(1) and myofibroblasts: a potential pathway towards renal scarring in human glomerular disease, Nephron 87 (2001), 240-248.

[6] R.C. Harris and E.G. Neilson, Toward a unified theory of renal progression., Апnи Rev Med 57 (2006), 365-380.

[7] N. Henke, R. Schmidt-Ullrich, R. Dechend, J.K. Park, F. Qadri, M. Wellner, M. Obst, V. Gross, R. Dietz, F.C. Luft, C. Scheidereit and D.N. Muller, Vascular endothelial cell-specific NF-kappaB suppression attenuates hypertension-induced renal damage, Circ Res 101 (2007), 268-276.

[8] M.A. Hernández-Presa, C. Gómez-Guerrero, J. Egido, In situ non-radioactive detection of nuclear factors in paraffin sections by Southwestern histochemistry, Kidney Int 55 (1999), 209214.

[9] T. Koji, K. Komuta, M. Nozawa, S. Yamada and P.K. Nakane, Localization of cyclic adenosine 3',5'-monophosphateresponsive element (CRE)-binding proteins by southwestern histochemistry, J Histochem Cytochem 42 (1994), 1399-1405.

[10] S.A. Mezzano, M. Barria, M.A. Droguett, M.E. Burgos, L.G. Ardiles, C. Flores and J. Egido, Tubular NF-kappaB and AP-1 activation in human proteinuric renal disease, Kidney Int $\mathbf{6 0}$ (2001), 1366-1377.

[11] S. Mezzano, C. Aros, A. Droguett, M.E. Burgos, L. Ardiles, C. Flores, H. Schneider, M. Ruiz-Ortega and J. Egido, NFkappaB activation and overexpression of regulated genes in human diabetic nephropathy, Nephrol Dial Transplant 19 (2004), 2505-2512.

[12] N. Miyata, Y. Gon, S. Nunomura, D. Endo, K. Yamashita, K. Matsumoto, S. Hashimoto and C. Ra, Inhibitory effects of parthenolide on antigen-induced microtubule formation and degranulation in mast cells, Int Immunopharmacol 8 (2008), 874-880.

[13] U. Panzen, O.M.Steinmetz, J.E. Turner, T.N. Meyer, G. Zahner, C. Gómez-Guerrero, R.M. Schmid, U. Helmchen, G.W. Moeckel, G. Wolf, R.A. Stahl and F. Thaiss, Resolution of renal inflammation: a new role for NF-kappaB1 (p50) in inflammatory kidney diseases, Am J Physiol Renal Physiol 297 (2009), 429-439.
[14] W. Qi, X. Chen, J. Holian, E. Mreich, S. Twigg, R.E. Gilbert and C.A. Pollock, Transforming Growth Factor - beta 1 differentially mediates fibronectin and inflammatory cytokine expression in kidney tubular cells, Am J Physiol 291 (2006), 1070-1077.

[15] G.K. Rangan, Y. Wang, Y.C. Tay and D.C. Harris, Early administration of PDTC in adriamycin nephropathy: effect on proteinuria, cortical tubulointerstitial injury, and NF-kappaB activation, Ren Fail 23 (2001), 773-780.

[16] G.K. Rangan, Y. Wang and D. Harris, NF-kappaB signalling in chronic kidney disease, Front Biosci 14 (2009), 3496-522.

17] A.B. Sanz, M.D. Sanchez-Nino, A.M. Ramos, J. A. Moreno, B. Santamaria, M. Ruiz-Ortega, J. Egido and A. Ortiz, NF-kB in Renal Inflammation, J Am Soc Nephrol 21 (2010), 12541262.

[18] G.E. Silva, R.S. Costa, R.C. Ravinal, M.A. dos Reis, M. Dantas and T.M. Coimbra, Mast cells, TGF-beta1 and alpha-SMA expression in IgA nephropathy, Dis Markers 24 (2008), 181190.

[19] S. Tamada, T. Nakatani, T. Asai, K. Tashiro, T. Komiya, T. Sumi, M. Okamura, S. Kim, H. Iwao, T. Kishimoto, S. Yamanaka, K. Miura, Inhibition of nuclear factor-kappaB activation by pyrrolidine dithiocarbamate prevents chronic FK506 nephropathy, Kidney Int 63 (2003), 306-314.

[20] C. Weigert, U. Sauer, K. Brodbeck, A. Pfeiffer, H.U. Häring and E.D. Schleicher, AP-1 proteins mediate hyperglycemiainduced activation of the human TGF-betal promoter in mesangial cells, J Am Soc Nephrol 11 (2000), 2007-2016.

[21] H.M. Wilson, S. Chettibi, C. Jobin, D. Walbaum, A.J. Rees and D.C. Kluth, Inhibition of macrophage nuclear factor-kappaB leads to a dominant anti-inflammatory phenotype that attenuates glomerular inflammation in vivo, Am J Pathol 167 (2005), 27-37.

[22] L. Zheng, R. Sinniah and S.I. Hsu, Renal cell apoptosis and proliferation may be linked to nuclear factor-kappaB activation and expression of inducible nitric oxide synthase in patients with lupus nephritis, Hum Pathol 37 (2006), 637-647. 


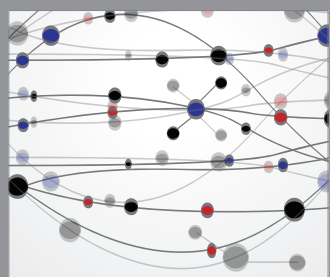

The Scientific World Journal
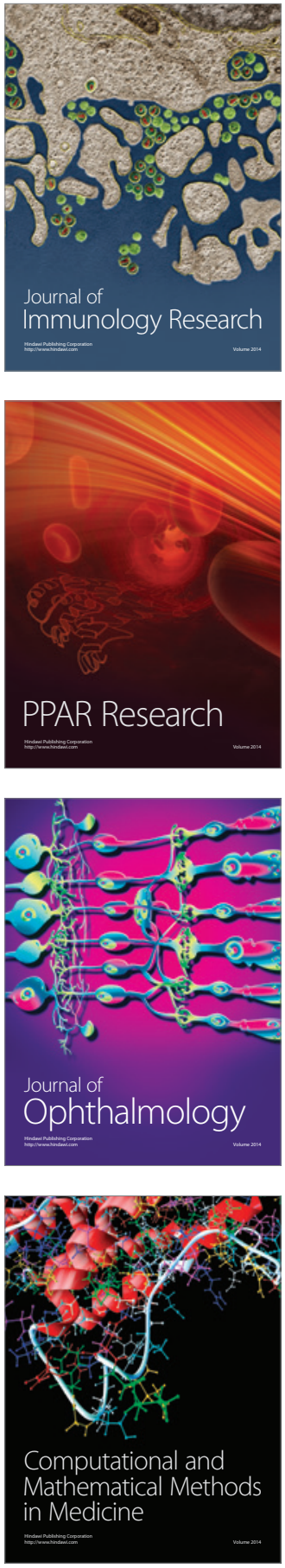

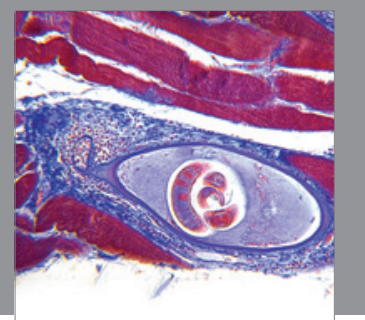

Gastroenterology

Research and Practice
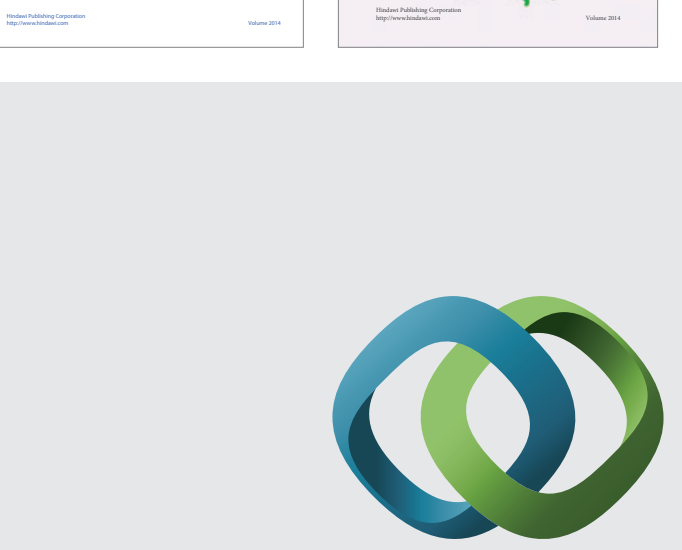

\section{Hindawi}

Submit your manuscripts at

http://www.hindawi.com
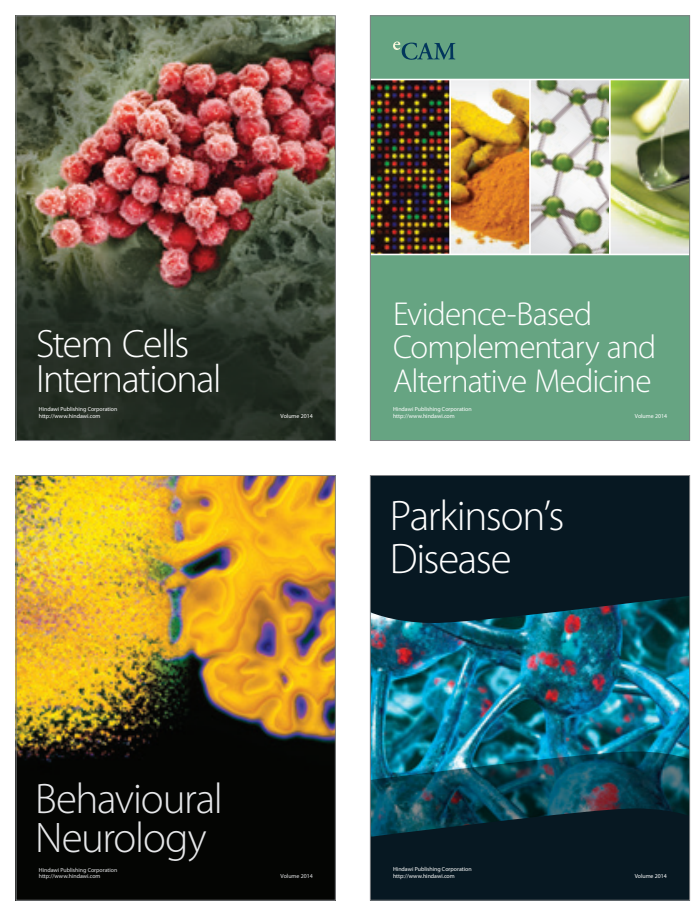

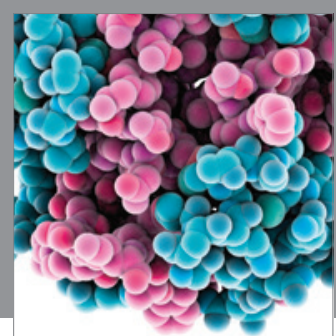

Journal of
Diabetes Research

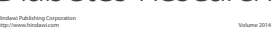

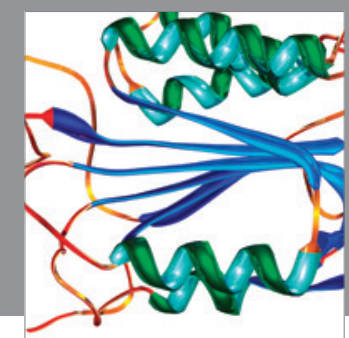

Disease Markers
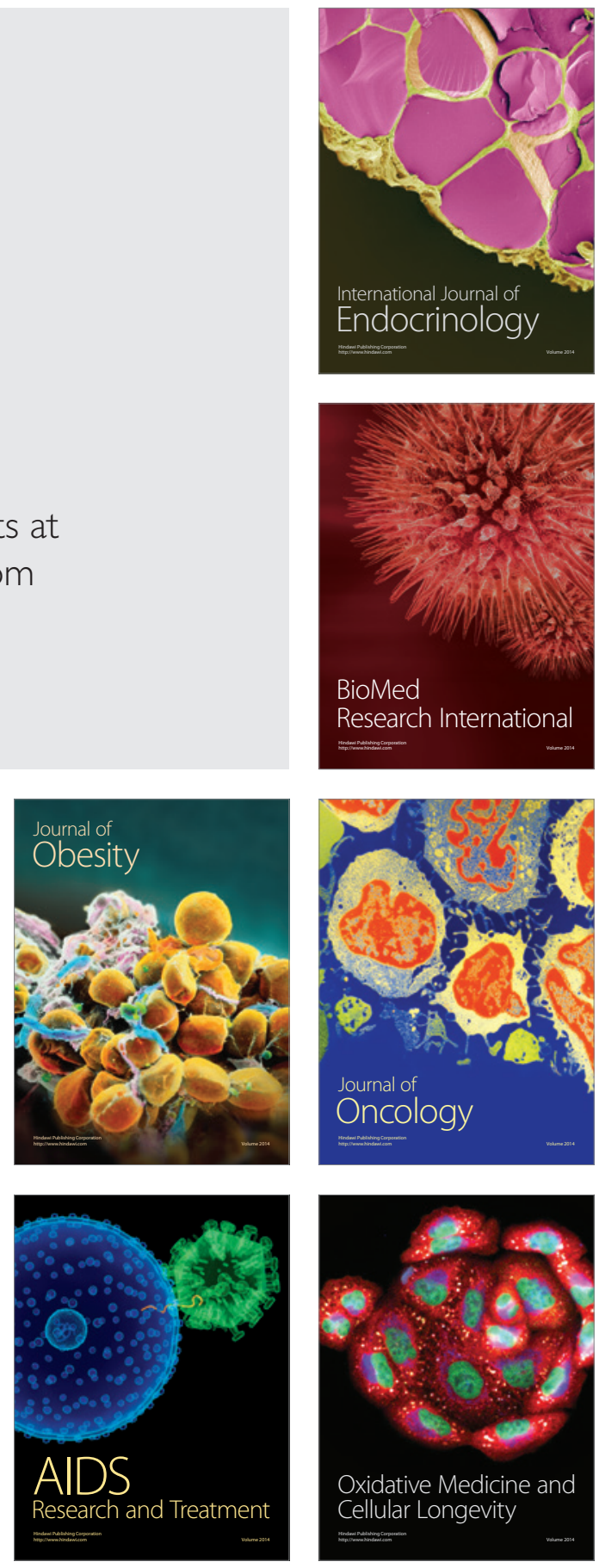